\title{
Paul Boulet, Pierre Devraigne et leur collection : témoins d'un siècle de wagnérisme en France
}

\section{Mathieu Schneider}

\section{Q OpenEdition}

1 Journals

Édition électronique

URL : https://journals.openedition.org/rbnu/2307

DOI : $10.4000 /$ rbnu.2307

ISSN : 2679-6104

Éditeur

Bibliothèque nationale et universitaire de Strasbourg

\section{Édition imprimée}

Date de publication : 1 mai 2013

Pagination : 20-29

ISBN : 9782859230432

ISSN : 2109-2761

\section{Référence électronique}

Mathieu Schneider, «Paul Boulet, Pierre Devraigne et leur collection : témoins d'un siècle de wagnérisme en France », La Revue de la BNU [En ligne], 7 | 2013, mis en ligne le 01 mai 2013, consulté le 08 août 2021. URL : http://journals.openedition.org/rbnu/2307 ; DOI : https://doi.org/10.4000/rbnu. 2307

\section{cc) (이}

La Revue de la BNU est mise à disposition selon les termes de la Licence Creative Commons Attribution - Pas d'Utilisation Commerciale - Partage dans les Mêmes Conditions 4.0 International. 


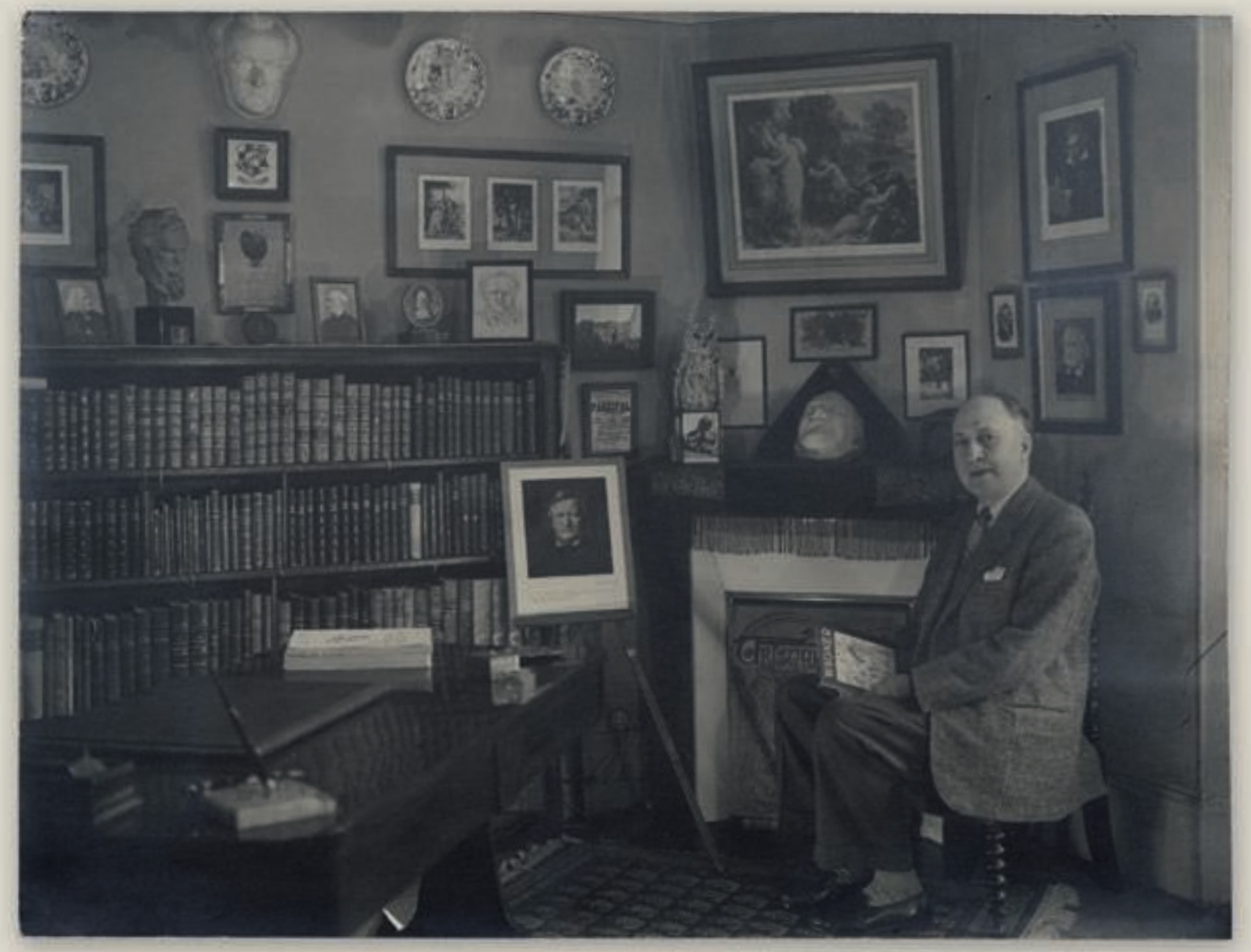

Paul Boulet dans les années 1950 dans son " musée Wagner ",

la pièce de son domicile de Vaucresson où il avait installé les objets de sa collection. On reconnaît, au mur, la série d'assiettes présentée dans la rubrique « Portfolio " (coll. BNU) 


\section{Paul Boulet, Pierre Devraigne et leur collection : témoins d'un siècle de wagnérisme en France}

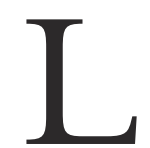

a Bibliothèque nationale et universitaire de Strasbourg s'est portée acquéreur, au cours de l'année 2012, d'un important fonds documentaire sur la réception de Richard Wagner en France, constitué d'un millier d'ouvrages, brochures et études, de près de six cents articles de journaux, d'une centaine de tableaux et illustrations représentant Wagner et ses œuvres, d'une trentaine d'autographes de membres de la famille Wagner et d'artistes, d'objets divers - souvent de dévotion - et de deux correspondances inédites entre l'un des collectionneurs et Winifred et Wieland Wagner. Ce fonds résulte de la réunion de deux collections : une première constituée par Paul Boulet, administrateur des douanes et fervent wagnérien, qui a rassemblé les quatre cinquièmes du fonds, vraisemblablement entre 1937 et 1970, et une seconde ajoutée à la première en 1972 par Pierre Devraigne, ancien maire de Paris, wagnérien passionné et fondateur du Cercle national Richard Wagner de Paris.

L'objet du présent article n'est pas de présenter en détail ce fonds ${ }^{1}$, qui sera partiellement exposé en juillet et août 2013 à la Stadtbibliothek de Bayreuth, en septembre à l'ambassade de France à Berlin et en octobre et novembre de la même année à la Médiathèque André Malraux de Strasbourg, mais d'en évaluer l'importance quantitative et qualitative pour l'histoire du wagnérisme en France. Cette question a une vraie pertinence, quand on sait que Wagner est le compositeur le plus documenté de l'histoire de la musique. Ce fonds apporte-t-il de nouvelles pièces ? Modifie-t-il les connaissances sur la réception de Wagner ? Témoignant des trois grandes périodes de l'histoire de la réception du compositeur en
France, quisontsuccessivementlapériodedel'émulation artistique (1861-1918), suscitée par les théories et les œuvres de Wagner, la période de marginalisation (19181945), liée à la récupération de Wagner par les nazis, et enfin la période de démythification (depuis 1945), visant à proposer une lecture plus moderne et décomplexée de Wagner, le fonds Boulet-Devraigne propose un regard diachronique sur le compositeur. Quelle est la nature de ce regard ? Quel est son point de mire ? Ce sont autant de questions auxquelles nous tenterons de répondre à partir de deux angles d'approche : celui des collectionneurs et celui de la collection. Celle-ci sera évaluée à l'aune de son originalité et de sa richesse pour l'histoire du wagnérisme, et les collectionneurs à la lumière du regard qu'ils ont porté sur Wagner et son œuvre.

\section{Paul Boulet (1884-1971), témoin et collectionneur}

On sait très peu de choses sur Paul Boulet, tout d'abord parce que ses descendants se sont rapidement départis de sa collection et ensuite parce que Pierre Devraigne, qui en a fait l'acquisition par l'intermédiaire d'un libraire parisien, ne savait lui non plus rien qu'il eût pu transmettre à ses enfants. A ce jour, nous n'avons donc pu retrouver aucun témoin qui fût en contact direct avec Boulet et qui aurait pu détenir des informations sur la manière dont s'est constituée cette importante collection. Les registres d'état civil $^{2}$ et la feuille de services ${ }^{3}$ de Boulet au ministère des Finances nous ont cependant fourni quelques informations tangibles qui, recoupées avec celles contenues dans le 
fonds, et notamment dans sa correspondance, nous permettent de formuler des conjectures fiables, du moins plausibles sur sa vie et son rapport à Wagner.

Pierre Paul Boulet naquit le 2 janvier 1884 à Brebières (Pas-de-Calais), soit un an à peine après la mort de Wagner et un an avant la création de la Revue wagnérienne. De sa jeunesse, on ne sait rien. A l'âge de dix-neuf ans, on le retrouve à Rouen comme surnuméraire de l'administration des douanes, dans laquelle il gravit patiemment les échelons, d'abord sur les côtes de la Manche (à Boulogne, à Dunkerque, puis au

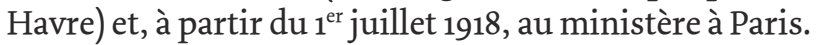
C'est là qu'il termina sa carrière, le 2 janvier 1944, avec le grade d'administrateur honoraire des douanes et décoré de plusieurs hautes distinctions, parmi lesquelles la Légion d'honneur dont il fut promu officier en 1936. Sa reconnaissance lui valut également d'assurer, pendant près de vingt ans, dans les années 1920-1930, des cours à la prestigieuse Haute école de commerce de Paris. Peu après sa retraite, Boulet quitta son domicile parisien du 10, avenue d'Iéna $\left(16^{\mathrm{e}}\right)$ pour emménager dans un pavillon en banlieue, à Vaucresson, où il installa sa collection dans ce qu'il appelait son " musée Wagner ». Il y mourut le 28 juin 1971.

Cette carrière dans les douanes eut au moins deux impacts sur la collection : elle donna tout d'abord à Boulet les moyens financiers ${ }^{4}$ pour acquérir l'ensemble des précieux ouvrages et illustrations qui la composent (plus de mille pièces), mais aussi pour effectuer, entre 1937 et 1970 (avec une interruption entre 1939 et 1950), le voyage à Bayreuth. Elle le poussa ensuite à s'intéresser particulièrement à la biographie d'Edmond Roche (18281861), le traducteur choisi par Wagner pour le Tannhäuser parisien de 1861 et natif du Pas-de-Calais comme Boulet. Ce dernier publia un premier article sur Roche en 1941, à l'occasion du $80^{\circ}$ anniversaire de Tannhäuser, dans les Annales des douanes, puis rédigea, sous forme de tapuscrit, une étude plus détaillée en 1951. Il fut également à l'origine d'une saynète, écrite par Madeleine Guignebert et Henri Wetzmann, intitulée Le douanier de Wagner, Edmond Roche et présentée à la RTF en mars 1961 dans l'émission "Soirée de Paris ». Tous ces documents ont bien entendu été versés à la collection.

On ne sait en revanche pas vraiment quand Boulet commença à se passionner pour Wagner, ni quand il se décida à débuter sa collection. Le descriptif sommaire de cette dernière, qui fut probablement rédigé par ses légataires pour le commissaire-priseur de la vente aux enchères de 1972, indique qu'elle fut rassemblée entre
1937 et 1970 , ce qui suppose que le premier voyage de Boulet à Bayreuth, en 1937, aurait été l'événement déclencheur. Toutefois, la collection contient près d'un demi-millier d'articles sur Wagner, ayant paru entre 1890 et 1960 (la plupart toutefois entre 1920 et 1960). Il est peu probable que ces articles aient tous pu être rassemblés après leur parution, si bien qu'on peut penser que Boulet avait commencé à s'intéresser à Wagner avant son premier voyage à Bayreuth, donc peu après son arrivée à Paris en 1918, et que le voyage le décida à entamer un véritable travail de collectionneur, patient et méticuleux.

Toutes les pièces de la collection rassemblée par Boulet portent un ex-libris que celui-ci fit confectionner à partir de l'insigne de la famille Wagner et sur lequel figure une date : 1941. Cette date est un élément clé pour comprendre le sens de la collection : en effet, 1941 marque à la fois le $80^{\mathrm{e}}$ anniversaire de la création française de Tannhäuser (et donc, dans un sens, la naissance du wagnérisme en France), la tenue d'une exposition sur Le Vaisseau fantôme, dont on fête le centenaire de la composition, dans la maison de Meudon où Wagner le composa ${ }^{5}$, et la première publication de Boulet sur Edmond Roche (dans les Annales des douanes). Le lien de Wagner à la France y est, dans les trois cas, central. Marquer la collection du sceau de cette année signifiait donc pour Boulet se donner l'ambition de documenter la réception de Wagner en France et le regard que les Français ont porté sur l'œuvre du compositeur.

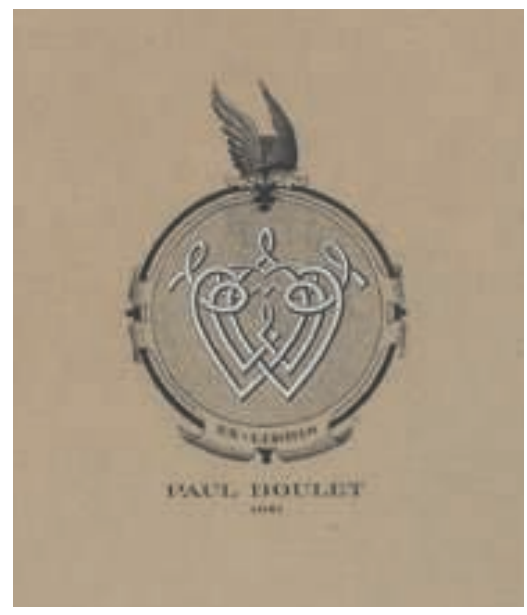

L'ex-libris de Paul Boulet, qu'il apposa sur tous les objets de sa collection (coll. BNU) 
Le fait que la collection prit corps en tant que telle à la fin des années 1930 n'est certainement pas un hasard. La montée du nazisme et les relations que la presse étalait au grand jour entre Hitler et la famille Wagner (notamment Winifred) ne pouvaient qu'interroger sur le sens profond de l'œuvre de Wagner et sa portée. Boulet se posait ces questions. Il semblait même sceptique par rapport à ce prétendu lien. Les commentaires qu'il fit par exemple en marge des ouvrages tendancieux de Houston G. Chamberlain montrent qu'il n'approuvait pas les positions antisémites et belliqueuses de l'Allemagne nazie et qu'il refusait de voir en Wagner une caution intellectuelle à ce régime.

La collection de Boulet répond donc clairement à la volonté de comprendre la signification profonde de l'œuvre de Wagner. Cela se traduit d'abord dans le choix des ouvrages acquis par lui : à côté des classiques sur le pèlerinage à Bayreuth, comme Lavignac ou Saint-Auban, à côté des grands textes critiques et polémiques de la naissance du wagnérisme (Judith Gautier, Charles Baudelaire, Adolphe Jullien, Edouard Dujardin, Catulle Mendès...), Boulet a rassemblé de nombreux ouvrages et articles qui expliquent la musique de Wagner, ou qui en proposent une indispensable traduction pour le public français, en général non germanophone. Figurent, parmi ces ouvrages, des textes célèbres comme ceux de l'Alsacien Edouard Schuré (Le Drame musical, en deux tomes), de Maurice Kufferath (analyses systématiques de tous les opéras de Wagner), de Joséphin Péladan (Le Théâtre complet de Wagner), de Georges Servières (Tannhäuser à l'Opéra en 1861), de J.-G. Prod'homme, le traducteur des écrits de Wagner; on en trouve de plus rares, comme ces articles de Louis Laloy (par exemple sur le drame musical moderne, article paru dans Le Mercure musical en 1905) ou de Jean du Tillet qui commenta plusieurs opéras de Wagner dans La Revue bleue entre 1895 et 1901. A chaque fois, c'est de musique qu'il est question : de la nomenclature des leitmotive, du rôle des instruments de l'orchestre, de la mélodie vocale. Tous ces articles et ouvrages étaient le plus souvent conçus comme des guides décryptant, pour l'auditeur profane ou pour le mélomane averti, la musique et le texte des opéras de Wagner. Ils s'apparentaient à des gloses de la Bible wagnérienne, visant à en démocratiser l'usage et à diffuser la bonne parole au plus grand nombre. Boulet dut y trouver de quoi satisfaire sa curiosité de nouveau converti au wagnérisme. Lorsqu’il pénétrait dans le temple du Festspielhaus, il pouvait déjà faire figure d'initié.

Boulet ne faisait pas partie de ces mélomanes qui possèdent des rudiments de théorie musicale : les commentaires qu'il a mis en marge des ouvrages de sa collection ne concernent toujours que le sens des opéras de Wagner et leur réception, jamais l'analyse musicale. En revanche, la mise en scène est un élément qui a beaucoup attiré son attention. La correspondance qu'il entretint entre 1951 et 1966 avec Wieland Wagner est à ce titre éloquente, car Boulet s'y montre très conservateur ${ }^{6}$. Il incarne clairement l'ancienne garde du public de Bayreuth d'aprèsguerre, qui était attachée à une fidèle observance des prescriptions scéniques de Wagner. Dans ses lettres, Boulet finit par en devenir obsessionnel et par réclamer que le " cadre" et le " lieu ", qu'il compose presque systématiquement en majuscules, fussent conformes aux didascalies de Wagner.

Enfin, Boulet céda aussi, comme beaucoup de collectionneurs, au fétichisme : on trouve donc dans la collection des autographes de la famille Wagner (une lettre de Wagner lui-même à un dénommé Siebert et des lettres ou billets de Cosima, Siegfried, Friedelind...), sans lien avec Wagner et la France. On trouve aussi tous les albums photos de Boulet à Bayreuth, patiemment légendés. Le collectionneur s'y présente toujours dans un face-à-face quasi narcissique avec le Maître : devant la tombe de Wagner, devant le buste de Wagner dans le parc du Festspielhaus, sur le parvis de la villa Wahnfried...

Comment situer Boulet dans l'histoire du wagnérisme ? Il en fut tout d'abord un témoin qui, conscient de l'importance de cette question en France, voulut léguer à la postérité une importante somme d'informations sur ce que Wagner a représenté dans ce pays, sur 
un plan artistique, culturel et politique. Sa longévité lui permit de vivre (et de couvrir) une période de près de cent ans, allant de la mort de Wagner au Bayreuth de Wolfgang ; elle fut aussi responsable du décalage générationnel dont souffrit Boulet après 1945 à Bayreuth. Du coup, il se retrouvait malgré lui en porte-à-faux avec les valeurs mêmes portées par le premier wagnérisme en France, qu'il avait si patiemment documenté : ce qui avait supporté l'émergence de nouveaux courants comme le symbolisme devenait, sous sa plume, le lieu du conservatisme et du passéisme. Au fond, n'était-ce pas aussi ce que représentait la musique de Wagner entre les deux guerres et après 1945 ?

\section{Pierre Devraigne (1913-1974), héritier et visionnaire}

Pierre Devraigne et Paul Boulet ne se sont pas connus. S'ils l'avaient fait, leur sujet de conversation eût été tout trouvé, mais tout laisse à penser que très vite, leurs discussions auraient abouti à une impasse. Devraigne appréciait les mises en scène de Wieland Wagner, que Boulet critiquait vertement. Leur rapport à la collection, en général, était très différent : Devraigne, s'il avait une passion profonde et sincère pour Wagner, n'était pas à proprement parler un collectionneur. Il a du reste quantitativement très peu enrichi la collection de Boulet, et surtout, les objets qu'il y a ajoutés sont plus des témoignages de ses séjours (officiels et privés) à Bayreuth, que le rassemblement patient et systématique d'objets dans un but précis. Devraigne était avant tout un homme politique, à l'aise en société, qui mit son amour pour Wagner au service d'une conviction politique et d'une générosité naturelle qui le poussèrent à faire partager à d'autres sa passion. Parler d'une " collection Boulet-Devraigne » est donc un abus de langage.

L'apport de Devraigne à la collection comprend essentiellement des médailles et divers objets honorifiques qu'il reçut à titre officiel à Bayreuth (Eichala, médailles et monnaies de la Ville, buste de Wagner...), des albums photos de ses voyages personnels et officiels à Bayreuth, des objets liés au Festival de Bayreuth (dont trois baguettes de chefs wagnériens prestigieux : Cluytens, Knappertsbusch et Böhm), des coupures de journaux documentant les relations entre Bayreuth et Paris, et des discours sous forme de tapuscrits ou d'enregistrements sonores. Si, numériquement, ces objets représentent peu au regard de ce que Boulet avait amassé, ils confèrent un tout autre sens à la collection de ce dernier. D'une relation individuelle à Wagner, presque sacrale, on découvre avec Devraigne combien la figure du compositeur eut aussi le pouvoir de fédérer : Devraigne fait toujours le pèlerinage à Bayreuth, accompagné de sa famille, d'amis ou de wagnériens de Paris. D'une vision française conservatrice de Wagner, le regard s'ouvre vers des interprétations plus modernes de l'œuvre du Maître. D'un Wagner dont Boulet défendait déjà qu'il pût (et peut-être dût) signifier autre chose que la discorde entre la France et l'Allemagne, Devraigne fit la figure d'une réconciliation entre les deux pays.

Devraigne appartenait à une nouvelle génération, celle de Wieland (dont il est de quatre ans l'ainé). Gynécologue-accoucheur de profession, né le $1^{\text {er janvier }}$ 1913, il s'était déclaré gaulliste dès les débuts de la guerre et avait rejoint la résistance. Il adhéra ensuite, dès sa création en 1947, au RPF (Rassemblement du peuple français), et siégea pendant un quart de siècle au Conseil municipal de Paris, dont il fut le président de mars 1959 à juin 196o. Il créa, en 1965, le Cercle national Richard Wagner, premier cercle Wagner à voir le jour dans un pays non germanophone, dont il resta président jusqu'à sa mort le 22 septembre $1974^{7}$.

Son mandat de président du Conseil municipal de Paris fut placé d'emblée sous le signe de la réconciliation franco-allemande et celle-ci, pour Devraigne, le wagnérien passionné, ne pouvait passer que par celui qui avait été, entre 1880 et 1918, le point focal des débats en France sur l'Allemagne, à savoir : le compositeur de Tannhäuser. C'est ès qualités qu'il se fit donc inviter, par le maire de Bayreuth Hans Walter Wild, au Festival de Bayreuth de 1959. Il ne vint pas les mains vides puisqu'il profita de l'occasion pour décorer, malgré les réticences du ministre des Affaires étrangères, le chef d'orchestre Hans Knappertsbusch de la Légion d'honneur, le 23 juillet 1959. Il remettra d'autres médailles par la suite, dont la médaille de la Ville de Paris à Karl Böhm en 1970. Devraigne était convaincu que la culture pouvait servir de ciment entre les peuples. Il l'affirma haut et fort lors du premier discours qu'il prononça dans les salons de l'hôtel de ville de Bayreuth, le 22 juillet 1959. " L'Art, dit-on, n'a pas de patrie [...] Paris, ville des arts par excellence, rend visite à Bayreuth, ville où souffle l'esprit. Paris, ville française, rend visite à Bayreuth, ville allemande. Cette rencontre a une signification, plus haute que celle, qui pourrait être banale, d'un président du Conseil municipal et d'un Oberbürgermeister. Il y a déjà cent ans, Richard Wagner, ce génie, l'avait compris et pressenti. Il nous appartient, maintenant que nous avons fait les 


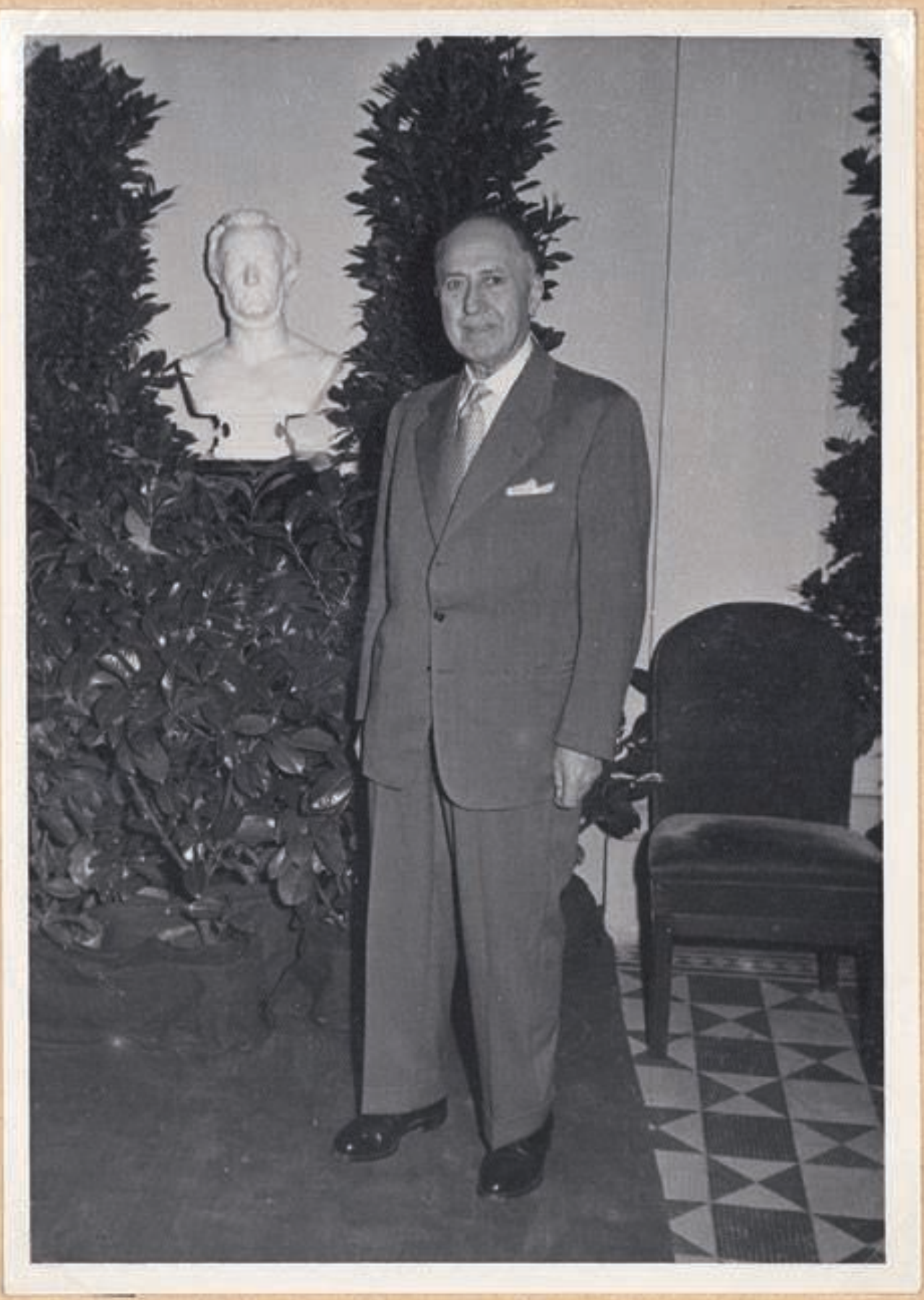

1955.- Bris du fuste de R. Wargner (Daus le vertiende dn Bestopielhous). 


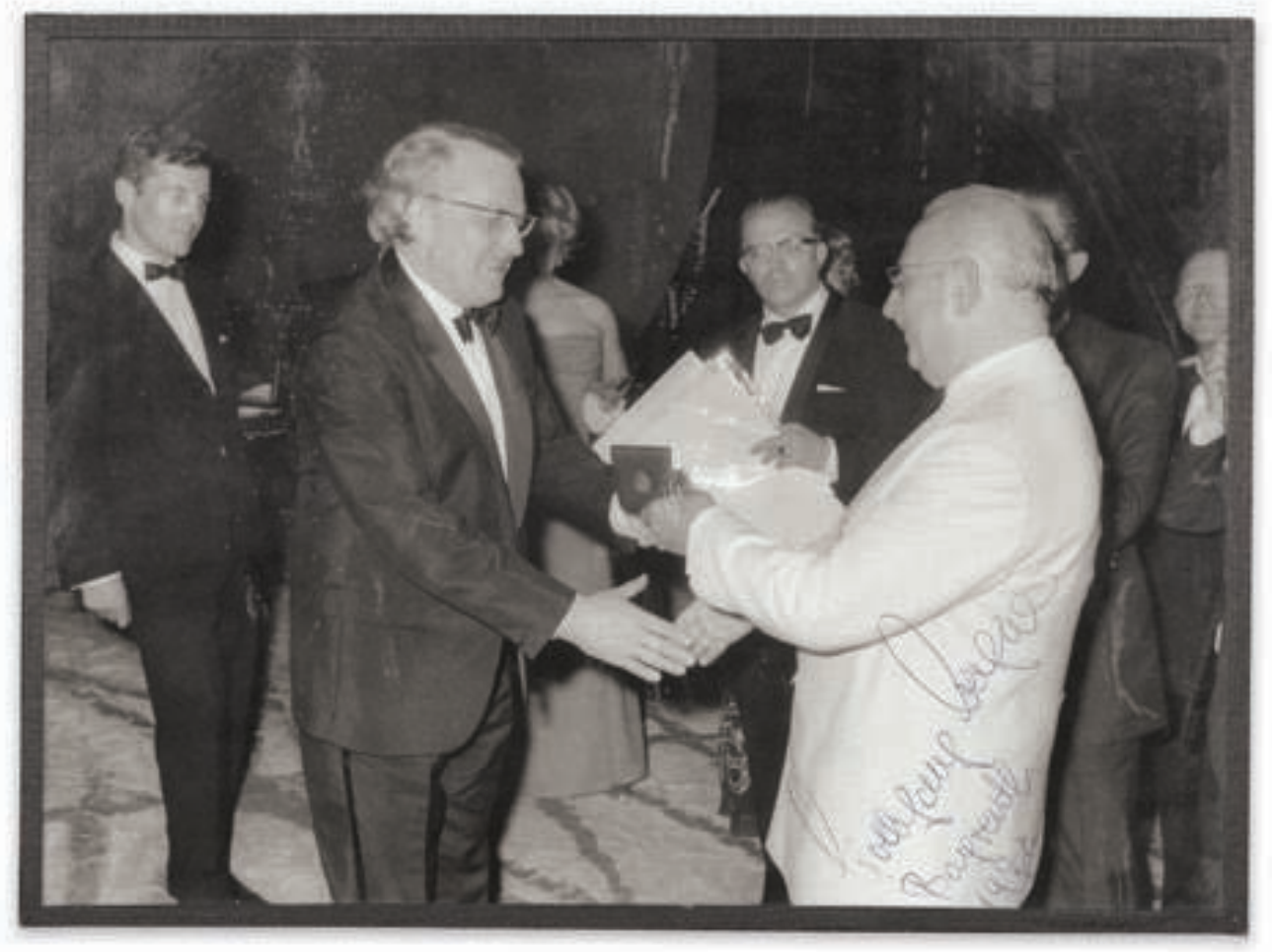

Pierre Devraigne remet la médaille de la Ville de Paris à Karl Böhm (Bayreuth, 27 juillet 1970 ; coll. BNU)

premiers pas les uns vers les autres, de faire de cette journée le point de départ de journées d'amitié et de compréhension $"{ }^{8}$. A l'axe diplomatique Paris-Berlin, Devraigne savait qu'il fallait adjoindre un axe culturel Paris-Bayreuth, et Wagner en devenait le point de mire.

Au-delà des relations officielles entre les deux villes, Devraigne eut rapidement l'ambition d'emmener les Parisiens à Bayreuth. C'est dans ce but qu'il fonda, le 17 décembre $1965^{\circ}$, le Cercle national Richard Wagner, pour créer un lieu de débats et de rencontres autour du compositeur. La collection Boulet fut donc enrichie de nombreux documents témoignant des premières années du Cercle : photos, programmes de concerts, articles de journaux et la collection complète de la revue Le Cygne, qui était le bulletin du Cercle...

Cela prouve que cet axe franco-allemand, qui guida en partie la vie et l'œuvre de Wagner, est bel et bien ce qui donne au fonds Boulet-Devraigne sa cohérence et son sens : Boulet en avait fait le thème culturel de sa collection ; Devraigne, sur un plan politique, ambitionnait de faire de Wagner la figure de réconciliation entre la France et l'Allemagne. Le fonds documente l'histoire du wagnérisme à travers ses trois périodes, mais surtout à travers deux générations : celle qui avait sacralisé Wagner et en avait fait une figure emblématique de la musique allemande et de l'Allemagne, et celle qui voulait le démythifier, pour mieux le réhabiliter. L'action de Devraigne sur un plan politique est à rapprocher de celle, artistique, de Wieland Wagner ou, deux ans après la mort de Devraigne, de celle de Boulez et de Chéreau.

Pierre Devraigne se posa donc comme héritier et visionnaire. Héritier, il le fut tout d'abord, au sens moral et physique du terme, de la collection de Boulet, mais également d'un siècle de wagnérisme en France. La création du Cercle national Richard Wagner était finalement l'aboutissement et la concrétisation de ces mouvements wagnériens qui, en France, avaient foisonné dans les milieux artistiques et culturels depuis la fondation de la Revue wagnérienne par Dujardin en 1885. Visionnaire, il le fut parce qu'il entendit donner une nouvelle direction à ces mouvements, une direction constructive et fédératrice. 


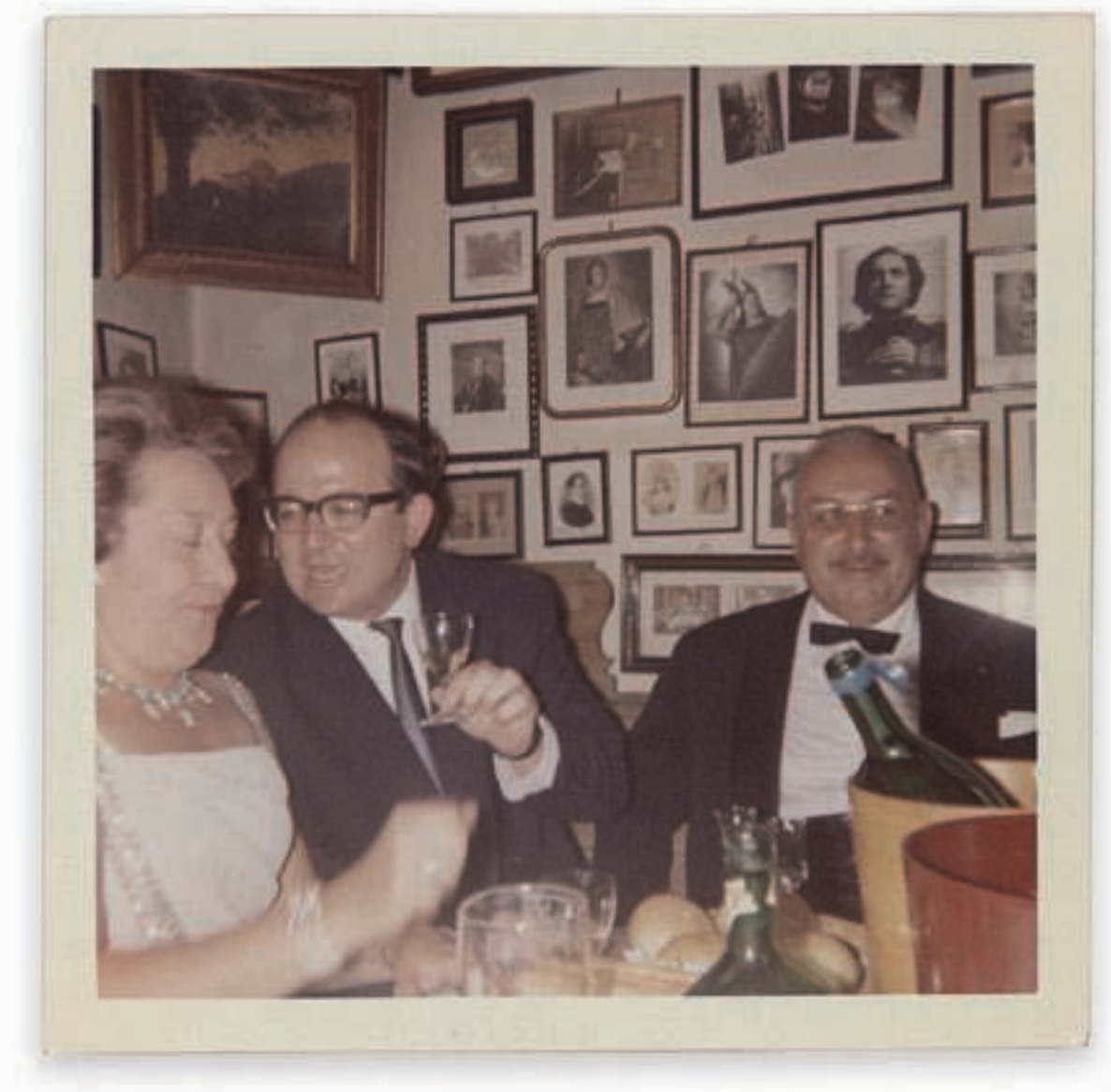

Pierre Devraigne à un souper au restaurant « Eule » de Bayreuth en août 1965 (coll. BNU) 


\section{L'apport du fonds Boulet-Devraigne à l'histoire culturelle du wagnérisme}

Même si toutes les pièces du fonds Boulet-Devraigne ne sont pas inédites, loin de là, le fonds, à plusieurs titres, présente un véritable intérêt documentaire pour l'histoire du wagnérisme. Tout d'abord, il a une valeur bibliophilique. Il documente de manière presque exhaustive les débats de la période d'émulation, tant au niveau littéraire que dans le domaine de la critique musicale, et ce par de belles éditions reliées, parfois dédicacées : à côté des premières éditions de Baudelaire, de Champfleury ou de Lavignac, on trouve ainsi de belles dédicaces, comme celle d'Adolphe Appia à Judith Gautier dans son livre La mise en scène du drame wagnérien (Paris, Léon Chailley, 1895), ou la mention autographe "À Mme Boulanger " de Gustave Fischbach dans son ouvrage De Strasbourg à Bayreuth (1882). Mais le plus précieux pour les passionnés de Wagner est ce demimillier d'articles sur le compositeur, parus entre 1890 et 1960. L'intérêt principal du fonds Boulet-Devraigne est en tout cas de réunir tous ces ouvrages et articles en un seul et même lieu.

La collection de Boulet permet également de jeter un nouveau regard sur les toutes premières années du wagnérisme, à travers le dossier des recherches de Paul Boulet sur Edmond Roche. Ce douanier, écrivain et poète à ses heures perdues, affichant une trentaine arrogante, entra en contact avec Wagner et lui proposa ses services pour la traduction et l'adaptation du livret de Tannhäuser en français. Son texte ne plut malheureusement pas au directeur de l'Opéra qui lui préféra la traduction de Charles Nuitter, mais la traduction de Roche était celle que Wagner avait choisie. Les recherches de Boulet apportent des informations et documents inédits (notamment la correspondance de Roche avec son administration, car Roche se fit porter pâle pour travailler avec Wagner) à cet épisode trop méconnu de l'histoire du wagnérisme.

Le fonds contient, sur la troisième période du wagnérisme, ces pièces inédites que sont les correspondances de Boulet avec Wieland et Winifred Wagner ${ }^{10}$, qui documentent le scandale bien connu des nouvelles mises en scène de Wieland, notamment celle des Maîtres chanteurs dans les années 1960. Mais elles confortent aussi nos connaissances sur les tiraillements au sein de la famille Wagner entre Winifred et ses fils, particulièrement Wieland.
L'intérêt du fonds Boulet-Devraigne ne serait pas complet s'il ne témoignait pas de toute la foisonnante création qui eut lieu autour de Wagner, notamment dans la première période du wagnérisme. La création littéraire est présente certes, mais plutôt discrète, notamment au travers de quelques volumes de Judith Gautier (Le Collier des jours) et des numéros de la Revue wagnérienne. En revanche, l'illustration wagnérienne est quantitativement bien plus représentée. Les lithographies de Fantin-Latour, dont certaines sont signées du peintre, en constituent la base pour la première période ; celles de Stassen ${ }^{11}$, pour la deuxième période, leur opposent une esthétique différente, surtout dans les planches consacrées à la Tétralogie qui exaltent les valeurs aryennes, plus que dans celles sur Tristan qui rappellent davantage la Sécession viennoise. Des illustrateurs moins connus, comme Ferdinand Leeke, Hermann Hendrich ou Jacques Wagrez, complètent utilement le fonds et dévoilent la palette d'esthétiques du tournant du $20^{\mathrm{e}}$ siècle : des plus progressistes (les françaises) aux plus conservatrices (souvent les allemandes). Devraigne, quant à lui, a enrichi la collection pour la troisième période des dessins de Clym.

Mais porter un jugement sur une collection, c'est aussi pointer ses manques. Si Boulet et Devraigne avaient pleinement voulu retracer l'histoire de la réception de Wagner en France, ils auraient dû joindre à leur collection les partitions et enregistrements de nombreuses œuvres composées à partir de Wagner, ou en réaction à Wagner en France. On aurait pu souhaiter y trouver les paraphrases de Messager, ou les opéras du wagnérisme comme Gwendoline de Chabrier ou Le Roi Arthus de Chausson. Car l'émulation créatrice du wagnérisme, surtout dans la première période (celle d'avant 1914), est centrale. Même si celle-ci a été plus intense en littérature qu'en musique, il est un peu dommage que la musique ait été oubliée. Cela traduit peut-être le goût avant tout littéraire des Français. Timothée Picard ne résumait-il pas le wagnérisme par la formule lapidaire : "Wagner, c'est la musique et l'Allemagne; Wagner, c'est la littérature et la France ${ }^{12}$ " ?

Enfin, l'histoire du wagnérisme en France, c'est aussi presque cent ans de discographie. Boulet, comme Devraigne, possédaient de nombreux disques vinyle. Les quelque 410 disques 78 tours que Boulet comptait chez lui n'ont malheureusement pas été intégrés au fonds lors de sa vente aux enchères; les très nombreux disques que possédait Devraigne n'ont eux non plus pas été versés à la collection, si bien que celle-ci, aujourd'hui, 
ne comprend presque aucun enregistrement. On mesurera la lacune que cela représente, tant d'un point de vue discographique que pour ce que ces disques nous auraient appris de leurs collectionneurs, qui plus est à une époque où le disque connut le boom le plus spectaculaire de son histoire. D'autres manques peuvent être constatés dans la collection, comme celui d'ouvrages qui prirent ouvertement parti contre Wagner (comme Le Coq et l'arlequin de Cocteau, ou bien la Poétique musicale de Stravinsky).

Le fonds Boulet-Devraigne a clairement pour ambition de documenter le débat sur l'Allemagne en France autour de Wagner, sur près de cent ans de son histoire. Boulet, le conservateur, collectionneur patient et méticuleux, en a écrit la thèse ; Devraigne, l'homme de conviction et d'ouverture, en rédigea quasiment l'antithèse. Il devait finalement appartenir à Catherine Devraigne, la fille de Pierre Devraigne qui, sur les conseils avisés de Philippe Olivier, proposa la collection à la BNU, d'esquisser la synthèse, en amenant le fonds à Strasbourg. Strasbourg est à la fois le symbole de la discorde entre Allemands et Français, pour qui elle fut, pendant soixante-quinze ans, le principal objet de convoitise, et le lieu de leur réconciliation. Elle est aussi une ville wagnérienne : sise sur les bords du Rhin, non loin de l'Odenwald où mourut Siegfried selon la légende, elle fut visitée à deux reprises par Wagner qui projeta un temps d'y créer Tristan. Elle regroupe aussi l'un des cercles Richard Wagner les plus actifs et les plus nombreux de France et peut s'enorgueillir d'avoir vu passer quelques disciples de Wagner, dont Gustav Mahler, Richard Strauss et Hans Pfitzner. Le fonds Boulet-Devraigne prend donc un sens tout à fait particulier à Strasbourg : il vient éclairer d'un jour nouveau, plus particulier, l'histoire de la ville. L'exposition qui s'y tiendra à l'automne sera donc pour le public alsacien une autre manière de voir et de concevoir les relations franco-allemandes.

\footnotetext{
Notes

1-Voir à ce sujet la présentation générale qui en est faite p. 104

2- Acte de décès $n^{\circ} 027$ de la commune de Vaucresson, établi le 2 juillet 1971

3- Ministère des Finances, feuille de service de Pierre Paul Boulet, n ${ }^{\circ} 1029$

4- L'appointement de Boulet, juste avant de prendre sa retraite en 1944, était de 90 ooo francs par an.

5- Exposition organisée par le Musée des Amis de Meudon-Bellevue du 25 mai au 3 novembre 1941. Boulet figurait parmi les invités au vernissage.

6- Voir à ce sujet la rubrique " L'inédit ».

7- Les informations sur la vie de Pierre Devraigne nous ont aimablement été communiquées, de vive voix, par Catherine et Patrice Devraigne, ses enfants, que nous tenons ici à remercier très chaleureusement.

8 - Pierre Devraigne, Au service de Paris. Discours de M. le Docteur Pierre Devraigne, Président du Conseil Municipal de Paris, Paris, Ville de Paris, s. d., p. 134. Nous remercions ici Catherine Devraigne de nous avoir prêté cet ouvrage.

9- Ceci est la date de parution de la création de l'association (loi 1901) au Journal officiel.

10 - Voir aussi la rubrique " L'inédit ».

11 - Voir aussi l'article sur Franz Stassen p. 88

12 - T. Picard, Wagner, une question européenne, Rennes, Presses universitaires de Rennes, 2006, p. 50
}

\section{Mathieu Schneider}

